\title{
Polarization Effects on Peptide Conformations at Water-Membrane Interface by Molecular Dynamics Simulation
}

\author{
PEDRO G. PASCUTTI ${ }^{1}$ KLEBER C. MUNDIM, ${ }^{2}$ AMANDO S. ITO, ${ }^{3}$ \\ PAULO M. BISCH ${ }^{1}$ \\ ${ }^{1}$ Instituto de Biofísica Carlos Chagas Filho, Universidade Federal do Rio de Janeiro, Ilha do Fundão, \\ 21949-900 Rio de Janeiro, Brazil \\ ${ }^{2}$ Departamento de Física, Universidade Federal da Bahia, Salvador, Brazil \\ ${ }^{3}$ Instituto de Física, Universidade de São Paulo, São Paulo, Brazil
}

Received 23 July 1998; accepted 9 February 1999

\begin{abstract}
The electrostatic image method was applied to investigate the conformation of peptides characterized by different hydrophobicities in a water-membrane interface model. The interface was represented by a surface of discontinuity between two media with different dielectric constants, taking into account the difference between the polarizabilities of the aqueous medium and the hydrocarbon one. The method consists of a substitution of the real problem, which involves the charges and the induced polarization at the surface of discontinuity, by a simpler problem formed with charges and their images. The electric field due to the polarization induced at the surface by charge $q$ was calculated using a hypothetical charge $q^{\prime}$ (image of $q$ ), symmetrically located on the opposite side of the surface. The value of $q^{\prime}$ was determined using the appropriate electrostatic boundary conditions at the surface. By means of this procedure, the effect of the interface can be introduced easily in the usual force field. We included this extension in the computational package that we are developing for molecular dynamics simulations (THOR). The peptides studied included hydrophilic tetraaspartic acid (Asp-Asp-Asp-Asp), tetralysine (Lys-Lys-Lys-Lys), hydrophobic tetrapeptide (His-Phe-Arg-Trp), an amphiphilic fragment of $\beta$-endorphin, and the signal sequence of the $E$. coli $\lambda$-receptor. The simulation results are in agreement with known experimental
\end{abstract}

Correspondence to: P. M. Bisch; e-mail: pmbisch@chagas.

biof.ufrj.br

Contract/grant sponsors: FAPESP; CAPES; FUJB-UFRJ 
data regarding the behavior of peptides at the water-membrane interface. An analysis of the conformational dynamics of the signal sequence peptide at the interface was performed over the course of a few nanoseconds. (C) 1999 John Wiley \& Sons, Inc. J Comput Chem 20: 971-982, 1999

Keywords: hydrophobic effect; molecular dynamics simulations; electrostatic image method; water-membrane model; $\beta$-endorphin and signal sequence conformations

\section{Introduction}

$\mathbf{T}$ he development of molecular modeling techniques is contributing to the understanding of biological processes at the atomic-molecular level and to the proposal of new molecular structures with high biological efficiency. Although the action of many drugs, hormones, and membrane proteins is dependent on the spatial conformation adopted by the molecules in the nonhomogeneous environment formed by the membrane and its neighborhood, in many cases the use of modeling techniques has been restricted to the simulation of single molecules, independently of the solvent medium. On the other hand, explicit simulations, atom by atom, of a system given by a polypeptide, water, and membrane molecules, are computerlimited. The monitoring of movements of a thousands-of-atoms assembly can be accomplished only during picoseconds or a few nanoseconds. ${ }^{1}$

An approach to simulate this interface using the electrostatic image method is possible by taking into account the difference between the polarizability of the aqueous medium and that of the hydrocarbon apolar one. ${ }^{2-4}$ This method has been applied to problems involving charges in the presence of conducting or dielectric discontinuity surfaces. Basically, it consists of the substitution of the real problem involving the charges and the induced polarization at the surface by a simpler problem comprised of charges and their images. The electric field produced by the charge density, induced by a charge $q$, at the surface of a conducting medium, or by the polarization induced at the surface of separation between two different dielectric media, is calculated as the field produced by a hypothetical charge, $q^{\prime}$ (image of $q$ ), symmetrically located on the opposite side of the surface. The value of $q^{\prime}$ can be determined precisely using electrostatic boundary conditions at the surface..$^{5}$

In the past, the image method was employed in the treatment of ion $^{3}$ and dipole $e^{4}$ monolayers at the interface between two immiscible fluids (aqueous and nonaqueous phases) to study the mechanical stability of this interface. Concerning the ion monolayer, its hydrodynamic instability at fluidfluid interface was pointed out to be relevant in biological phenomena, like cellular fusion and phagocytosis. ${ }^{3,4}$ This approach was based on an earlier model of ion monolayers at the water-membrane interface, where the counterions in the aqueous phase were treated using the image method. ${ }^{6}$ Within this model the aqueous solution was considered a perfect conducting medium, corresponding to a high concentration of counterions located close to the interface. An improvement of this model was proposed by considering that a fraction of the counterions is distributed in a Gouy-Chapman diffuse layer in the aqueous phase. ${ }^{7}$ The modification of the image charges due to this screening effect related to diffuse layers was considered in monolayer stability analyses., 4

We present an application of the electrostatic image method to molecular dynamics simulations of peptides containing $N$ partially charged atoms in the presence of an uncharged water-membrane interface. In the present approach, surface charge and diffuse layer effects are not taken into account, but there is an important contribution from the induced polarization at the separation between membrane and water phases. To control all calculations and to perform modifications where necessary, we developed a computational package for molecular optimization and dynamics simulations $\left(\mathrm{THOR}^{2,8}\right)$, based on the GROMOs force field. ${ }^{9}$ In the choice of potential energy function, we achieved a compromise between simplicity and accuracy, with flexibility to adapt different terms to the force field.

The main advantage of this method is that it allows simulations in long time scales (up to 100 ns) with ordinary computer facilities, providing a good description of physicochemical and conformational properties of the peptides, as will be discussed. In this study we describe the method and apply it to peptides with different hydropho- 
bicities. A hydrophilic behavior is expected for tetraaspartic acid (Asp-Asp-Asp-Asp), due to the four negative charges, as well as for tetralysine (Lys-Lys-Lys-Lys), which bears four positive charges. On the other hand, a hydrophobic behavior is expected for the His-Phe-Arg-Trp tetrapeptide. This sequence is the main one responsible for biological activity of the melanotropic peptide $\alpha$ MSH (melanocyte-stimulating hormone). ${ }^{10,11}$ In addition, it was also observed that it interacts with the lipid phase of vesicles. ${ }^{12}$

Many biologically active peptides, without stable secondary or tertiary structures in aqueous medium, could be stabilized in the presence of water-membrane interfaces, where their activity is expressed. ${ }^{13}$ Several peptides have hydrophilic residues forming a domain facing the aqueous medium and hydrophobic residues located at the opposite domain facing the apolar medium. This behavior was observed in amphiphilic $\alpha$-helices, either at the surface of proteins ${ }^{14,15}$ or at the surface of biological membranes. ${ }^{13}$ A specific human $\beta$-endorphin fragment, an opioid peptide of 31 residues presenting activity in membranes, shows a periodicity in the amino acid sequence that allows the formation of opposite hydrophobichydrophilic domains, characteristic of amphiphilic helices, between the residues 13 and 29. Taylor and Kaiser $^{13}$ proposed a model peptide to investigate the functional characteristics of the amphiphilic $\alpha$-helix based on the natural $\beta$ endorphin peptide. We studied this peptide, $\mathrm{H}_{2} \mathrm{~N}$-Pro-Leu-Leu-Lys-Leu-Leu-Gln-LysLeu-Leu-Leu-Gln-Lys-Leu-Phe-Lys-Gln-Lys$\mathrm{Gln}-\mathrm{OH}$, simulating it in the presence of the water-membrane interface model.

Signal sequences are peptides containing 15-36 residues, mainly hydrophobic, found in the amino terminal of proteins. These sequences assist the insertion of proteins into membranes or their translation across membranes. These peptides present no conformational preference in an aqueous medium, whereas a tendency for $\alpha$-helix formation was detected in apolar media. ${ }^{16-19}$ It was postulated that the stability of the helix would be an extra requirement for the biological signaling function. ${ }^{17,20}$ We studied here the signal sequence of the E. coli $\lambda$-receptor $\left(\mathrm{NH}_{3}-\mathrm{Met}-\mathrm{Met}-\mathrm{Ile}-\right.$ Thr-Leu-Arg-Lys-Leu-Pro-Leu-Ala-Val-AlaVal-Ala-Ala-Gly-Val-Met-Ser-Ala-Gln-AlaMet-Ala-COO). We have shown previously that the insertion of this peptide into the membrane depends on factors like charge distribution and initial conformation. ${ }^{2}$ Furthermore, we have also observed that a stable helix conformation was attained only after insertion of the peptide into the membrane. In this study, we monitored the stability of the helix at the interface during a 4-ns molecular dynamics simulation.

\section{Methods}

\section{ELECTRIC POTENTIAL DUE TO A POINT CHARGE IN THE PRESENCE OF AN INTERFACE BETWEEN TWO DIFFERENT DIELECTRIC}

The atomic-molecular polarization originates both from the atom and chemical bond electronic displacement and from the orientation of polar molecules in response to an electric field. This electric field, and consequently the induced polarization, could be produced by an externally applied field or by the presence of neighboring molecules. For one water molecule, the resulting dipole moment changes from 1.85 to 2.50 Debye when passing from the gas phase to the liquid phase. ${ }^{21}$ In an external electric field the aqueous medium dielectric constant, $\varepsilon$, is about 80 , due mainly to the orientation polarizability of the water molecules. ${ }^{22}$ On the other hand, apolar solvent dielectric constants are only about 1.5 or 2.0 , as a sole consequence of the electronic polarization of the molecules in an external field. There is no orientation contribution because of the absence of a resultant dipole moment in these molecules. ${ }^{23}$

The polarization of a dielectric medium depends on the atomic-molecular properties of the medium, and on the intensity of the applied external field. Assuming that the macroscopic response of an isotropic solvent to an applied field is linear if the intensity of the field is low, the macroscopic polarization, $\mathbf{P}$, is parallel to the external field, $\mathbf{E}$ :

$$
\mathbf{P}=\chi_{e} \mathbf{E}
$$

where $\chi_{e}$ is the electric susceptibility of the medium. The macroscopic electric field, represented by the electric displacement vector, $\mathbf{D}$, will have two contributions, one from the external field, E, and the other from the polarization, $\mathbf{P}$, of the medium ${ }^{5}$ :

$$
\mathbf{D}=\varepsilon_{0} \mathbf{E}+\mathbf{P}
$$

From eqs. (1) and (2), we have:

$$
\mathbf{D}=\varepsilon_{0} \mathbf{E}+\chi_{e} \mathbf{E}=\left(\varepsilon_{0}+\chi_{e}\right) \mathbf{E}=\mathscr{E} \mathbf{E}
$$

where $\varepsilon_{0}$ and $\mathscr{E}$ are the vacuum and material permissivity, respectively. $\mathscr{E}$ is related to the dielec- 
tric constant by $\varepsilon=\mathscr{E} / \varepsilon_{0}$. For all equations we used the MKSA unit system, consistent with GROMOS force field parameters.

A charge $q$ at a distance $d$ from a surface separating two media, with dielectric constants $\varepsilon_{1}$ and $\varepsilon_{2}$, respectively, polarizes the surface with a polarization charge density that can be represented by a fictitious charge $q^{\prime}$, image of $q$, located at the same distance $d$ on the other side of the surface. ${ }^{5}$ If the charge $q$ is located in medium 1, of dielectric constant $\varepsilon_{1}$, the electric potential $\left(\Phi_{1}\right)$ in this medium has contributions from the real charge $q$ and from the polarization charge given by the image $q^{\prime 5}$ :

$$
\Phi_{1}=\frac{1}{4 \pi \varepsilon_{0} \varepsilon_{1}}\left[(q / r)+\left(q^{\prime} / r^{\prime}\right)\right]
$$

where $r=\left[(x-d)^{2}+y^{2}+z^{2}\right]^{1 / 2}$ and $r^{\prime}=[(x+$ $\left.d)^{2}+y^{2}+z^{2}\right]^{1 / 2}$.

The electric potential $\Phi_{2}$ in medium 2, of dielectric constant $\varepsilon_{2}$, due to a real charge located in medium 1 , is calculated by replacing the real charge $q$ by an effective charge $q^{\prime \prime}$, in order to consider the polarization effects on the dielectric discontinuity surface ${ }^{5}$ :

$$
\Phi_{2}=\frac{1}{4 \pi \varepsilon_{0} \varepsilon_{2}}\left[\left(q^{\prime \prime} / r\right)\right]
$$

The exact values of $q^{\prime}$ and $q^{\prime \prime}$ are obtained using the appropriated boundary conditions at the surface. The potentials $\Phi_{1}$ and $\Phi_{2}$ must satisfy two boundary conditions at the discontinuity surface located at $x=0$ :

- First continuity of the electrostatic potential:

$$
\Phi_{1}=\Phi_{2} \quad \text { at } x=0
$$

- Second continuity of the normal component of the electric displacement:

$$
\varepsilon_{1}\left[\partial \Phi_{1} / \partial x\right]=\varepsilon_{2}\left[\partial \Phi_{2} / \partial x\right] \quad \text { at } x=0
$$

This last equation was obtained from eq. (3) and from the relation $\mathbf{E}=-\nabla \Phi$.

When expressions (4) and (5) are replaced into the boundary conditions (6) and (7), one obtains two coupled equations for $q$ and $q^{\prime}$, yielding the following solution:

$$
\begin{aligned}
& q^{\prime}=q\left[\left(\varepsilon_{1}-\varepsilon_{2}\right) /\left(\varepsilon_{1}+\varepsilon_{2}\right)\right] \text { and } \\
& q^{\prime \prime}=q\left[2 \varepsilon_{2} /\left(\varepsilon_{1}+\varepsilon_{2}\right)\right]
\end{aligned}
$$

Replacing these expressions back into eqs. (4) and (5), we obtain the potentials $\Phi_{1}$ and $\Phi_{2}$ as a function of $q^{5}$ :

$$
\begin{gathered}
\Phi_{1}=\frac{1}{4 \pi \varepsilon_{0} \varepsilon_{1}} q\left[(1 / r)+\left(\varepsilon_{1}-\varepsilon_{2}\right) /\left(\varepsilon_{1}+\varepsilon_{2}\right) r^{\prime}\right] \\
\Phi_{2}=\frac{1}{4 \pi \varepsilon_{0}} 2 q /\left(\varepsilon_{1}+\varepsilon_{2}\right) r
\end{gathered}
$$

We note that the image charges are introduced as a tool to calculate the electric field. Using the boundary conditions, we obtain the exact solution for the electric field created by one charge, both in the phase where the charge is located and in the adjacent phase, corrected by the polarization effects as given by eqs. (9) and (10), respectively.

\section{ELECTROSTATIC ENERGY FOR PAIR OF CHARGES AT MEMBRANE-WATER INTERFACE}

Because of factors like solubility and stability, the aqueous soluble proteins have, generically, a hydrophobic core circumvented by a hydrophilic region. The apolar residues are, in general, located in the interior of the protein and the polar or charged residues are found on the surface in contact with the polar solvent. The differences between the dielectric constant in the interior of these proteins and the aqueous solvent lead to the model of a cavity in a dielectric continuum and to the use of the electrostatic image method for their description. A limitation of the method, as noted by Friedman, ${ }^{24}$ Rogers, ${ }^{25}$ and van Gunsteren and Berendsen, ${ }^{1}$ is based on the fact that the approximation of one charge to the interface leads also to the approximation of the image charge-consequently producing a pole in the surface potential. This problem is even worse for aqueous soluble proteins because the charges of these macromolecules are generally found at their surfaces.

In our approach we do not consider direct interactions with the solvent, so that each charge will not experience the reactive field generated by its own image. Thus, the divergence in the electrostatic potential energy at the interface between the aqueous medium and the membrane is prevented. Each charge, $q_{i}$, experiences the field generated by all $q_{j}$ charges of the molecule and by their corresponding $q_{j}^{\prime}$ images, excluding the first and second chemically bound neighbors. In this way, one renormalizes the intramolecular electrostatic inter- 
actions under the effect of the polarization of the water-membrane interface.

In fact, the self-induced polarization will contribute to the total free energy changes when the peptide goes from the aqueous to the hydrocarbon phase. Therefore, other contributions with an important and possibly opposite effect, like the entropic contribution of hydration and other shortrange forces, are also not considered in the present effective medium approach. Hence, we are dealing with a partial contribution to the hydrophobic effect, and we cannot offer conclusions about the real partitioning of the peptide between the two phases. However, as will be discussed later, our model is in agreement with the general hydrophobic-hydrophilic behavior expected for the peptides considered. On the other hand, we expect that our model would give a good description of the possible conformations adopted by the peptides at the interface, because the most important aspects of the intramolecular forces have been considered through the renormalized electrostatic interactions.

The electrostatic energy between a pair of charges $q_{i}$ and $q_{j}$ is related to the electrostatic potential $\Phi$ through:

$$
V=q_{i} \Phi_{j}=q_{j} \Phi_{i}
$$

where $\Phi_{j}\left(\Phi_{i}\right)$ is the electric potential due to charge $q_{j}\left(q_{i}\right)$ at the position of the charge $q_{i}\left(q_{j}\right)$. Considering a pair of charges $q_{i}$ and $q_{j}$ immersed in a medium with dielectric constant $\varepsilon_{1}$, in the presence of an interface between this medium and another of dielectric constant $\varepsilon_{2}$, the electrostatic potential energy according to eq. (9) is given by:

$$
\begin{aligned}
V_{i j}= & \frac{1}{4 \pi \varepsilon_{0} \varepsilon_{1}} q_{i} q_{j} \\
& \times\left[\left(1 / r_{i j}\right)+\left(\varepsilon_{1}-\varepsilon_{2}\right) /\left(\varepsilon_{1}+\varepsilon_{2}\right) r_{i j}^{\prime}\right]
\end{aligned}
$$

where $r_{i j}$ is the distance between charges $q_{i}$ and $q_{j}$, and $r_{i j}^{\prime}$ the distance between charges $q_{i}$ and $q_{j}^{\prime}$.

If the pair of charges is immersed in the medium of dielectric constant $\varepsilon_{2}$, the electrostatic energy corresponds to:

$$
\begin{aligned}
V_{i j}= & \frac{1}{4 \pi \varepsilon_{0} \varepsilon_{2}} q_{i} q_{j} \\
& \times\left[\left(1 / r_{i j}\right)+\left(\varepsilon_{2}-\varepsilon_{1}\right) /\left(\varepsilon_{1}+\varepsilon_{2}\right) r_{i j}^{\prime}\right]
\end{aligned}
$$

In the case where the charges of the pair are in different media, separated by the interface, using eq. (10), we have, for the electrostatic potential energy:

$$
V_{i j}=\frac{1}{4 \pi \varepsilon_{0}} 2 q_{i} q_{j} /\left(\varepsilon_{1}+\varepsilon_{2}\right) r_{i j}
$$

Taking into account the effect of water-membrane interface polarization in the force field expression, the usual Coulomb potential term is replaced by eqs. (11), (12), or (13), depending on the position of the pair of charges $q_{i}$ and $q_{j}$ with respect to the interface.

We initially tested the model applying it to two hypothetical molecules, one containing a pair of equal charges $(+0.25$ and +0.25$)$ and the other a pair of opposite charges $(+0.25$ and -0.25$)$. The charges were aligned parallel to the interface and the distance between them was fixed at $4.1 \AA$. We calculated, for each molecule, the potential energy profile at the interface model using eqs. (11), (12), and (13), for $\varepsilon_{1}=80$ and $\varepsilon_{2}=2$. We obtained the energy values at different distances from the surface, on both sides of it. These results were compared with the energy obtained using the usual Coulomb term considering a homogeneous phase with the corresponding dielectric constant.

Figures 1 and 2 show that the method prevents discontinuity in the potential energy, smoothing the potential across the surface between the two media. Because the charges do not interact with their self-images, there is no divergence in the electrostatic potential energy when going through the interface. Far from the surface, the potential energy tends to the value of the corresponding homogeneous phase. Smoothing the potential across the interface can be understood by considering the contribution of images to the potential energy. In fact, from eq. (8) we can see that, if the charges are immersed in medium 1 , with a larger dielectric constant $\left(\varepsilon_{1}>\varepsilon_{2}\right)$, the image charges $q_{j}^{\prime}$ have the same sign as the real charges $q_{j}$. On the other hand, if the charges are in medium 2, with a lower dielectric constant, charges $q_{j}$ and $q_{j}^{\prime}$ have opposite signs [eq. (8)]. For a pair of charges of the same sign, this effect increases the electrostatic energy if the pair is in the high dielectric constant medium $\left[\varepsilon_{1}-\varepsilon_{2}>0\right.$ in eq. (11)], or decreases the electrostatic energy for the charges in the lower dielectric constant phase $\left[\varepsilon_{2}-\varepsilon_{1}<0\right.$ in eq. (12)], when compared with the electrostatic energy without polarization corrections (Fig. 1). In contrast, for a pair of opposite charges, the images contribute to the negative energy in eqs. (11) and (12), leading to an energy decrease when in the higher dielectric constant phase and to an increase when in the 


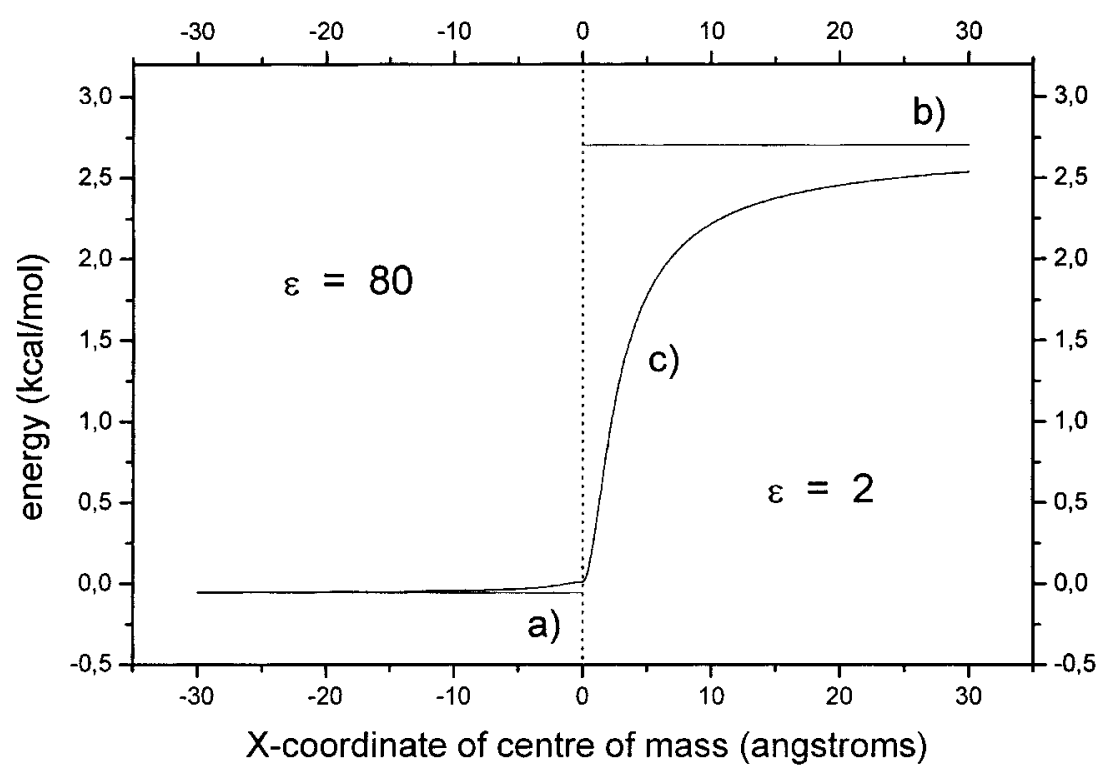

FIGURE 1. Interaction energy profile of a pair of equal charges: (a) in a solvent of $\varepsilon=80$; (b) in a solvent of $\varepsilon=2$; and (c) with corrections from the induced polarization.

lower dielectric phase (Fig. 2). If the charges of the test molecule are on different sides of the interface, the effective potential energy is obtained from the mean value of the dielectric constants according to eq. (13). We can show that the energy obtained from eq. (13) has exactly the same value as the limit obtained from eqs. (11) or (12), when one of the charges approaches the surface, assuring the continuity of the electrostatic energy profile.
Thus, when going from medium 1, with high dielectric constant, to medium 2, with low dielectric constant, either a pair of positive or a pair of negative charges goes over a potential barrier, whereas a pair of opposite charges goes down a potential barrier. In the environment with lower dielectric constant the potential curve is steeper due to the larger electrostatic interaction. At a distance of about $15 \AA$, one order of magnitude

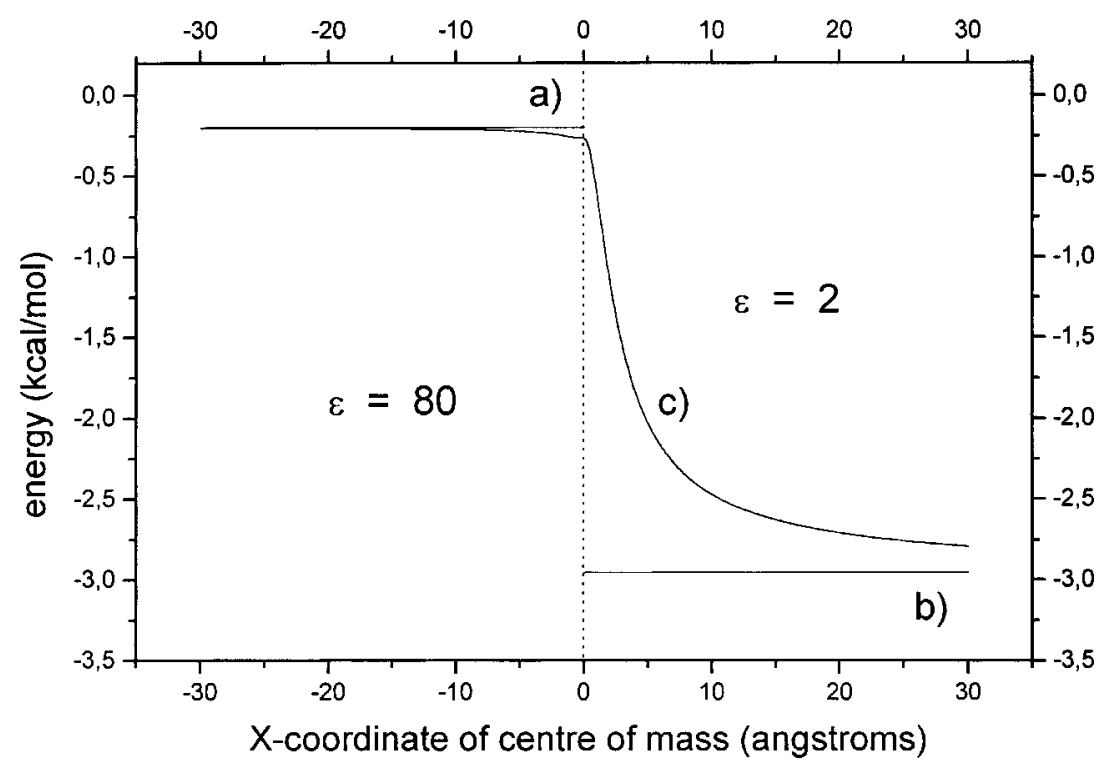

FIGURE 2. Interaction energy profile of a pair of opposite charges: (a) in a solvent of $\varepsilon=80$; (b) in a solvent of $\varepsilon=2$; and (c) with corrections from the induced polarization. 
higher than the length of a $\mathrm{C}-\mathrm{C}$ bond, the effect of the interface polarization is still meaningful in the low dielectric constant medium (Figs. 1 and 2).

As can be inferred from the gradient of the potential curves in Figures 1 and 2, there is a resulting net force acting on the molecules caused by the induced polarization charges at the separating surface between the two media. A pair of either positive or negative charges immersed in the higher dielectric constant medium is repelled by the surface polarization charges, whereas the same pair immersed in the lower dielectric constant medium is attracted. On the contrary, a pair of opposite charges in the higher dielectric constant medium is attracted by the surface polarization charges and, in the lower dielectric constant, is repelled. The resulting force on a macromolecule, in the presence of an interface between an aqueous phase and a hydrocarbon medium, will be the sum of the contributions over all pairs of nonbonded atomic charges. The force direction will be perpendicular to the interface and its orientation will depend on the distribution of the partial charges over the macromolecule.

\section{GENERALIZATION OF MOLECULAR FORCE FIELD}

The general potential energy function:

$$
\begin{aligned}
V\left(\left\{\mathbf{r}_{i}\right\}\right)= & V\left(\mathbf{r}_{1}, \mathbf{r}_{2}, \ldots, \mathbf{r}_{N a t}\right) \\
= & \sum_{n=1}^{N_{b}} \frac{1}{2} K_{b_{n}}\left(b_{n}-b_{0 n}\right)^{2} \\
& +\sum_{n=1}^{N \theta} \frac{1}{2} K_{\theta_{n}}\left(\theta-\theta_{0 n}\right)^{2} \\
& +\sum_{n=1}^{N \xi} \frac{1}{2} K_{\xi_{n}}\left(\xi_{n}-\xi_{0 n}\right)^{2} \\
& +\sum_{n=1}^{N \varphi} K \varphi_{n}\left[1+\cos \left(n_{n} \varphi_{n}-\delta_{n}\right)\right] \\
& +\sum_{i<j}^{N_{a t}}\left[C_{12}(i, j) / r_{i j}^{12}-C_{6}(i, j) / r_{i j}^{6}+V_{i j}\right]
\end{aligned}
$$

was used where the three first terms on the righthand side correspond to harmonic potentials describing the $N_{b}$ chemical bonds of the molecule, the $N_{\theta}$ angles between pairs of consecutive bonds, and the $N_{\xi}$ improper dihedral angles, respectively. The fourth term corresponds to the tortional poten- tial that describes the $N_{\varphi}$ dihedral proper angles. The last term corresponds both to the van der Waals potential and the Coulomb $V_{i j}$ term, describing the interaction among all pairs of atoms $i$ and $j$, excluding the first and second bound neighbors, whose interactions are described by the harmonic terms. We used the Gromos force field parameters. ${ }^{9}$ Because of the interface, the electrostatic term $V_{i j}$ includes the corrections using eqs. (11), (12), or (13), according to the relative position of the pair of charges with respect to the dielectric discontinuity surface.

\section{MOLECULAR DYNAMICS SIMULATIONS}

The tetrapeptides Asp-Asp-Asp-Asp, Lys-Lys-Lys-Lys, and His-Phe-Arg-Trp were acetylated to prevent the effect of charges in the terminals. The backbone dihedral angles of the first two peptides were set initially in a trans conformation $\left(\phi=\psi=\omega=180^{\circ}\right)$. The third peptide, starting from a random conformation, was submitted to an annealing procedure, by heating the molecule to $900 \mathrm{~K}$ and cooling to $0 \mathrm{~K}$, in 5000 steps of molecular dynamics, in an electrostatic continuum with $\varepsilon=80$. This procedure was repeated until a globular compact conformation was obtained. A more open conformation was also used to investigate the conformational effect of the peptide in the presence of the water-membrane interface.

The initial conformation for the $\beta$-endorphin fragment in the $\alpha$-helix was obtained from manipulation of the internal coordinates of the peptide, using the values of $-60^{\circ},-40^{\circ}$, and $180^{\circ}$ for the backbone dihedral angles $\phi, \psi$, and $\omega$, respectively. For the signal sequence peptide, we adopted the initial conformation proposed in the literature, based on nuclear magnetic resonance (NMR) studies that suggest an $\alpha$-helix from Leu8 to Met24. ${ }^{20}$

The geometry of the peptides was optimized with the steepest descent algorithm. ${ }^{26}$ Differences of less than $10^{-5} \mathrm{kcal} / \mathrm{mol}$ in the total energy between two successive steps were used to stop energy minimization. Molecular dynamics simulations were started at the reference temperature of $10 \mathrm{~K}$, with initial atomic velocities obtained from a Maxwell-Boltzmann velocity distribution, followed by heating to $300 \mathrm{~K}$ in $30 \mathrm{ps}$, using the leapfrog algorithm, ${ }^{27}$ in steps of $0.5 \mathrm{fs}$. During the simulations the velocities were rescaled every 50 steps if the temperature of the system was out of the limits of $\pm 10 \%$ from the reference temperature. 


\section{Results}

The simulations with the tetrapeptides were run initially in a continuum with $\varepsilon=80$, with the center of mass velocity maintained equal to zero, during a period of $200 \mathrm{ps}$, to equilibrate the system. These thermally stabilized conformations were then placed in the presence of the watermembrane interface.

The dynamics for the $\beta$-endorphin fragment started with the peptide already placed with the axis of the helix parallel to the interface, with the predominantly hydrophilic surface immersed in water $(\varepsilon=80)$, whereas the hydrophobic surface was in the nonpolar $(\varepsilon=2)$ effective medium.

The molecular dynamics for the signal sequence started in the continuum with $\varepsilon=2$, with the center of mass at a fixed position. After a short heating time, a period of $50 \mathrm{ps}$ was simulated to stabilize the system before the introduction of the water-membrane interface. To avoid the disappearance of the helix conformation, we introduced extra improper dihedral potentials over the peptide backbone, with high force constants fixing the angular positions corresponding to the characteristic $\alpha$-helix values. After a $100-p s$ simulation in the presence of the interface the constraints were suppressed and the conformational stability was monitored. $^{2}$

For each molecule we monitored the trajectories of the center of mass, following the $x$-coordinate along the axis perpendicular to the interface. The surface of separation between the two media was placed at $x=0$. The trajectories were compared with the potential energy profiles obtained from optimized molecular structures with the center of mass positioned along the $x$-axis, at intervals of $0.5 \AA$, on both sides of the discontinuity surface (Fig. 3).

For the signal peptide, we followed the backbone dihedral angles to monitor the conformational changes during a 4-ns simulation (see Fig. 6).

\section{TETRAPEPTIDES AND $\beta$-ENDORPHIN FRAGMENT}

Figure $3 a$ shows the trajectories of both tetrapeptides and the $\beta$-endorphin fragment center of mass. The trajectories follow the potential energy profiles obtained by energy minimization of each structure along the $x$-axis perpendicular to the interface (Fig. 3b-d). The trajectories of the charged peptides confirm their affinity for the polar environment, once they move from the interface and are immersed in the interior of the medium with $\varepsilon=80$ (Fig. 3a). The potential energy profile of tetraaspartic acid agrees with its trajectory, presenting a large barrier for the entry of the peptide into the membrane. On the other hand, the energy profile for tetralysine shows a small minimum at the interface, inside the membrane. This finding agrees with the attribution of an amphiphilic character to the side chain of lysine, due to the long hydrocarbon chain with a positive charge at the extremity in neutral $\mathrm{pH} .{ }^{13}$

The $\beta$-endorphin fragment, as expected, has an amphiphilic behavior. Initially, its trajectory fluctuates between positions 5 and $10 \AA$, inside the membrane. It stabilizes at circa. $5 \AA$ from the dielectric discontinuity surface after about 150 ps (Fig. 3a). Its potential energy profile presents two minima, one at $10 \AA$ and the lowest at $5 \AA$ (Fig. 3c). A potential energy barrier of about 100 $\mathrm{kcal} / \mathrm{mol}$ confines the amphiphilic peptide to the interfacial region. The optimized structure of the peptide before dynamics shows a compact and linear helix (Fig. 4a). The stabilized structure at the interface is less uniform due to thermal fluctuations, and a deviation from the helical form is observed in the C-terminal (Fig. 4b). This could be attributed to helix instability in the terminal regions, usually observed in isolated peptide fragments. However, the preservation of the helix conformation over a large extent of the molecule could be assigned to the stabilizing forces present at the interface, associated with the deep potential well in this region. These stabilizing forces would also be present on the surface of proteins.

The trajectory of the His-Phe-Arg-Trp peptide shows that it sinks into the hydrophobic interior of the membrane (Fig. 3a). The potential profiles for the two initial conformations studied here present lower energies at $\varepsilon=2$, characterizing their hydrophobic behavior (Fig. 3d). Both conformations are illustrated in Figure 5 in a van der Waals surface representation. In the medium of $\varepsilon=80$, the open structure has a lower energy when compared with the packed conformation; on the contrary, in the $\varepsilon=2$ medium, the packed structure has the lower energy (Fig. 3d). These results suggest that the partitioning of the peptide in the membrane would be accompanied by a conformational rearrangement.

It has been discussed in the literature that, for some peptides, an extended conformation can be 
a)

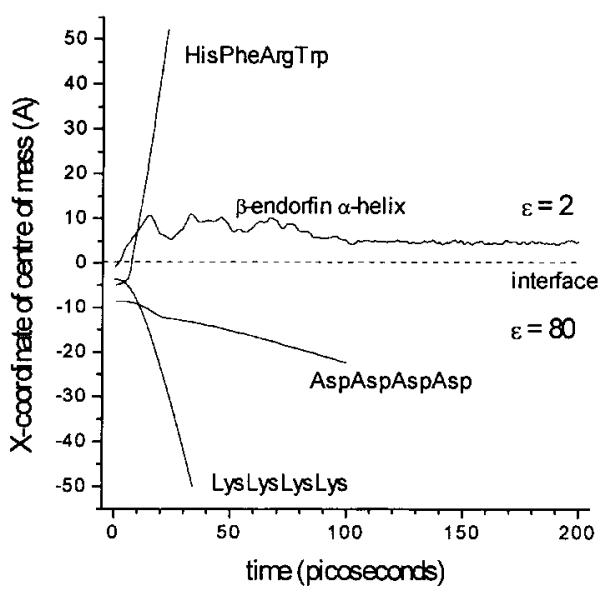

c)

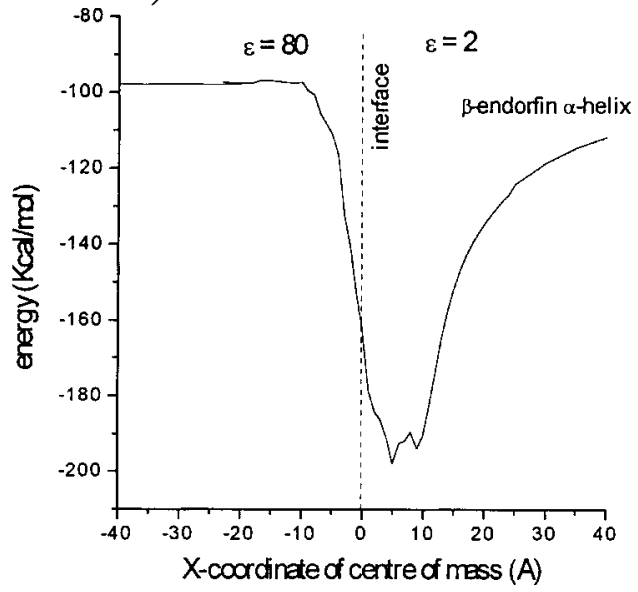

b)

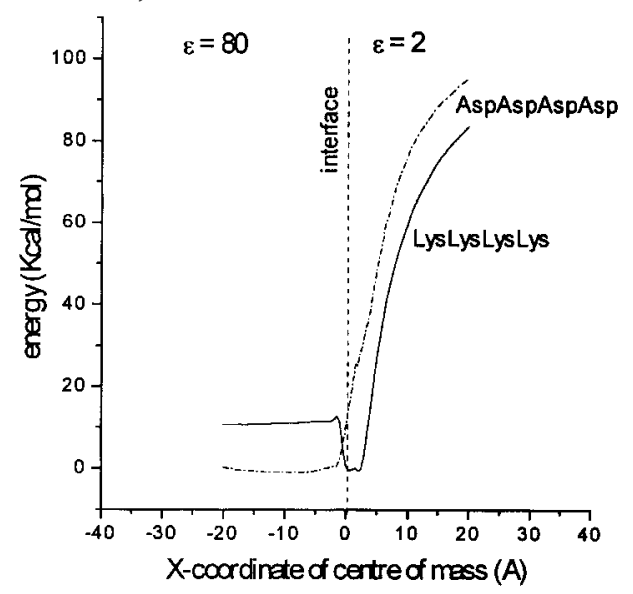

d)

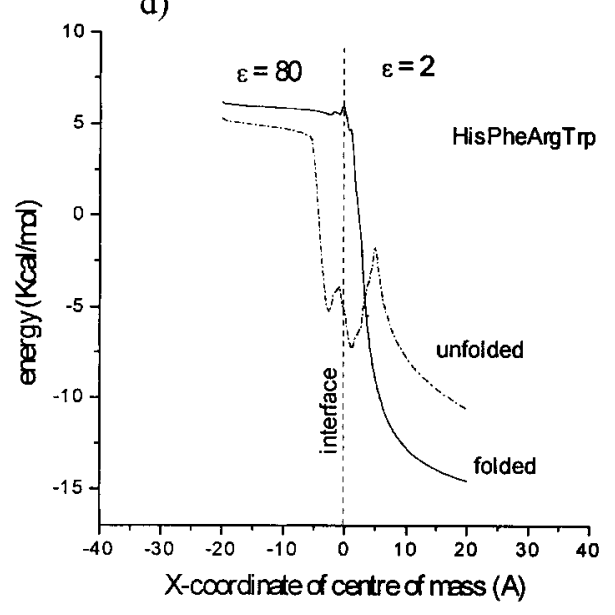

FIGURE 3. (a) Time course of the center of mass of the tetrapeptides and the $\beta$-endorphin model. Potential energy profile through the interface: (b) for Asp-Asp-Asp-Asp and Lys-Lys-Lys-Lys; (c) for the $\beta$-endorphin fragment; and (d) for two different conformations of His-Phe-Arg-Trp.

expected in water. ${ }^{13}$ We have shown that the peptides can be packed and stabilized in a low dielectric constant medium like the membrane phase. Furthermore, the interface between the aqueous solution and the membrane could play a role in the conformational changes of peptides, as can be deduced by comparing the potential profile of folded and unfolded peptides in Figure 3d. Although the potential energy of the unfolded state is lower inside the membrane, there is a potential barrier at the interface, which would prevent the entrance of the peptide in this phase. In all likelihood, the conformational changes are required to cross this barrier, or even to evolve to the most favorable energy of the folded state. The large potential well for the endorphin fragment in a helix conformation and the stable conformation acquired by this peptide during the dynamics simulation show that the electrostatic interfacial forces are quite important for the conformational behavior of this peptide at the membrane-water interface (Fig. 3a and c).

\section{SIGNAL SEQUENCE}

It has been proposed that the signal sequence of the $\lambda$-receptor (and some of its mutants) presents an affinity for lipid phases, and that they are able to form $\alpha$-helices in organic solvents. ${ }^{28}$ Infrared, circular dichroism, and NMR spectroscopy studies indicate that the functional sequences have a strong preference for secondary structures in $\alpha$-helix conformations. ${ }^{20,29,30}$ We have previously shown ${ }^{2}$ that the signal sequence peptide was captured in a 


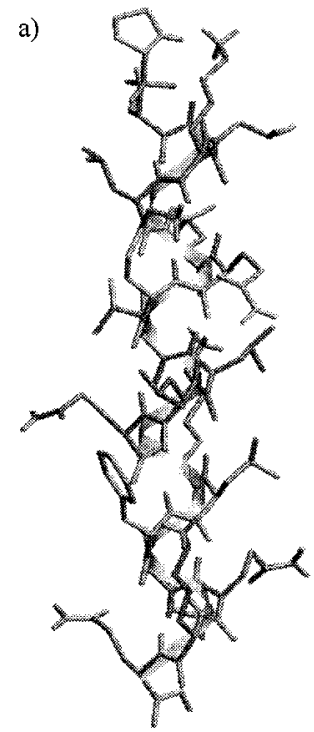

b)

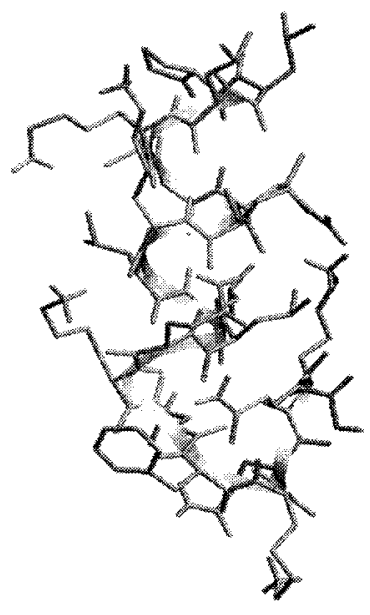

FIGURE 4. Molecular structure of the $\beta$-endorphin fragment at the membrane-water interface: (a) initial optimized $\alpha$-helix conformation; and (b) thermal stabilized structure after 200 ps of molecular dynamics simulation at $300 \mathrm{~K}$.

potential well at around $10 \AA$ from the interface, and that the majority of the residues in the $\alpha$-helix maintained the structure during 50 ps of dynamics in that position.

We have monitored, during several nanoseconds, the dihedral $\phi$ and $\psi$ angles from Leu8 to Met24. These residues correspond to the region that maintains the $\alpha$-helix conformation. ${ }^{20} \mathrm{We}$ observed a conformational instability in the helix between Leu8 and Val12, probably because of the proline residue (Pro9), which usually interrupts $\alpha$-helix propagation (Fig. 6a). The peptide became bent in this region, remaining stabilized by salt bridges between the C-terminal and the N-terminal, and between the C-terminal and residues Arg6 a)

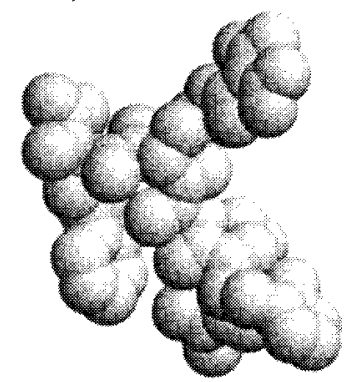

b)

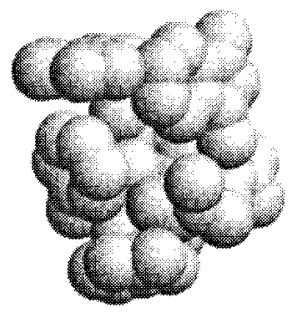

FIGURE 5. Representations of the: (a) open; and (b) packed conformations used in the simulations of the His -Phe-Arg-Trp peptide. or Lys7, as suggested before. ${ }^{2}$ In the region between residues 8 and 12 there were some conformational interconversions between metastable secondary structures, usually with a lifetime of $>1$ ns (Fig. 6a). We also observed that the conformational transitions occurred in short time intervals in a cooperative process. For example, at time $t \cong 1500 \mathrm{ps}$, all five residues simultaneously changed their conformation (Fig. 6a).

From Ala13 to Ala21, the helix conformation remained stable, with higher flexibility in Met19 and Ala21 residues. The other residues remained more or less rigid, including the glycine residue (Gly17), which usually makes the peptide chain more flexible (Fig. 6a and b). As expected, the C-terminal region (residues 22-24) is more flexible, because, in this region, the stabilization of the secondary structures by the hydrogen bond cooperative effect is reduced considerably (Fig. 6b). The residue Gln22 remained in a $\beta$-turn conformation for a long time, probably as a consequence of the salt bridges formed with the C-terminal.

Fluorescence studies of this signal sequence containing tryptophan in different positions suggests that the helix region remained immersed in lipid bilayers, and that these peptides probably have a transmembrane character. ${ }^{31,32}$ We verified that the center of mass of the molecule stabilizes at $10 \AA$ apart from the interface, with the turn in the $\mathrm{N}$-terminal exposed to the hydrophilic medium and the helix portion immersed in the hydrophobic one, perpendicular to the interface. This result is illustrated in Figure 7, where the peptide backbone images recorded from the simulations are shown at intervals of $1 \mathrm{~ns}$ during a simulation of 4 ns.

We observed that the region conserved in the helix is smaller than that proposed by Bruch and Gierasch. ${ }^{20}$ However, we studied the peptide in the presence of the water-membrane interface, whereas the experimental results were obtained in a continuous solvent. On the other hand, the presence of a second interface, considering that the membrane has a finite width, could lead to a different potential profile for the molecule, eventually resulting in different behavior of the helix segment.

\section{Conclusions}

The hydrophobic effect has enthalpic and entropic contributions, coming from solute-solvent, solvent-solvent, and solute-solute interactions, as 

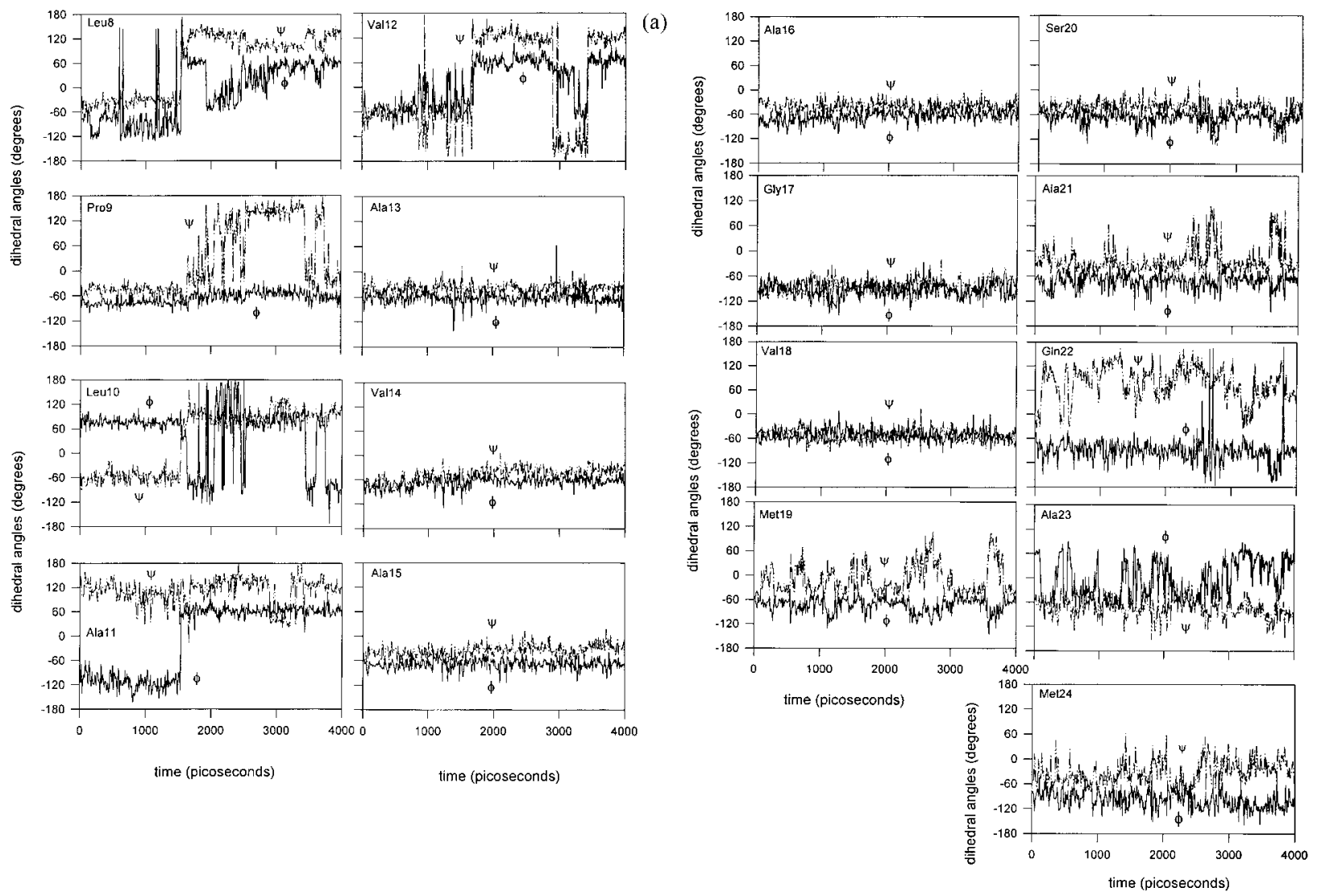

FIGURE 6. (a) Time evolution of the dihedral angles $\phi$ and $\psi$ of Leu8 to Ala15 and (b) Ala16 to Met24 residues of the $\lambda$-receptor signal sequence.

well as from internal rearrangements in the solute and solvent molecules. Even considering only a partial contribution to the total effect, we have shown that the hydrophobic, hydrophilic, or amphiphilic behavior of the peptides studied is

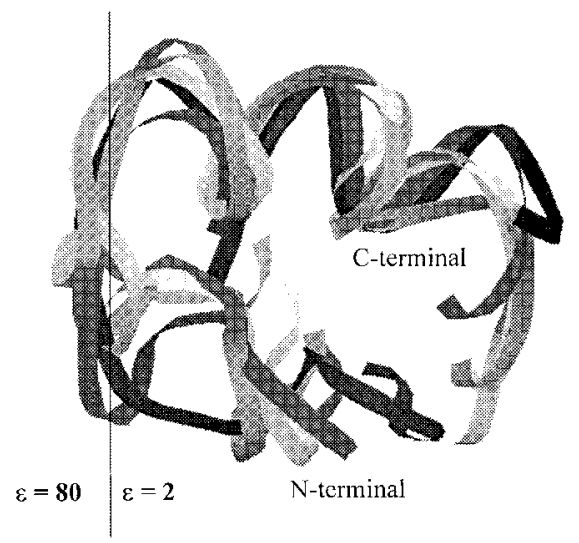

FIGURE 7. Superposition of signal sequence backbone images taking at each 1-ns interval in a dynamic simulation of $4 \mathrm{~ns}$. reproduced by renormalizing the electrostatic interactions, treating the polarization of the water-membrane interface with the method of electrostatic images. This means that such an electrostatic effect is an important contribution to the partitioning of the molecules, at least for the peptides considered here. We have also demonstrated that the polarization forces could drive the movements of the peptides. Furthermore, these forces are responsible for the stabilization of specific conformations of the peptides at the interface. As a consequence, we can expect that bioactive peptides without a secondary structure in aqueous solution present well-defined conformations at biological interfaces stabilized by the polarization forces discussed here.

Using the present method, we have shown that a very simple approach can describe the main features of the conformational changes of peptides at a membrane-water interface. The model is based on very well-established physical principles. Further corrections, such as for example taking into account a continuous variation of the dielectric 
constant across the interface or the incorporation of extra potentials describing further hydrophobic and self-polarization effects, would involve the disadvantage of introducing unknown parameters. These parameters should be adjusted, and modifications on the actual simple form of the force field function would be necessary. In the context of the present molecular dynamics methods it seems that the only consistent and reasonable extension of this approach would be the explicit representation of all water and membrane molecules. However, this procedure involves very high computational cost. Even using supercomputers, or parallelization, it is generally only possible to simulate molecular dynamics of picosecond order, (in reasonable CPU time). A possible solution strategy is to use a simple model, like in the present approach, to investigate dynamics in the nanosecond timescale, and the explicit solvent representation for more detailed investigation in the picosecond timescale.

\section{Acknowledgments}

This work received computational support from the Nucleo de Computação Electrônica (UFRJ). We acknowledge Ms. Fernada S. Leite for revising the English.

\section{References}

1. van Gunsteren, W. F.; Berendsen, H. J. C. Angew Chem Int Ed Engl 1990, 29, 992.

2. Arêas, E. P. G.; Pascutti, P. G.; Schreier, S.; Mundim, K. C.; Bisch, P. M. J Phys Chem 1995, 99, 14882.

3. Bisch, P. M.; Van Lamsweerde-Gallez, D.; Sanfeld, A. J Coll Interf Sci 1979, 71, 501.

4. Van Lamsweerde-Gallez, D.; Bisch, P. M.; Sanfeld, A. J Coll Interf Sci 1979, 71, 513.

5. Reitz, J. R.; Milford, F. J.; Christy, R. W. Foundations of Electromagnetic Theory; Addison-Wesley: Reading, MA, 1967; Jackson, J. D. Classical Electrodynamics; Wiley: New York, 1962.

6. Miller, C. A.; Scriven, L. E. J Coll Interf Sci 1974, 46, 477.

7. Mingins, J.; Pethica, B. A. Trans Faraday Soc 1963, 59, 1893.

8. Moret, M. A.; Pascutti, P. G.; Bisch, P. M.; Mundim, K. C. J Comput Chem 1998, 19, 647.
9. van Gunsteren, W. F.; Berendsen, H. J. C. Groningen Molecular Simulation (GROMOs) Library Manual; Biomos: Groningen, 1987.

10. Hruby, V. J.; Wilkes, B. C.; Cody, W. L.; Sawyer, T. K.; Hadley, M. E. Peptide Prot Rev 1984, 3, 1.

11. Hruby, V. J.; Sharma, S. D.; Toth, K.; Jaw, J. Y.; Al-Obeidi, F.; Sawyer, T. K.; Hadley, M. E. In: Vaudry, H.; Eberle, A. N., eds. The Melanotropic Peptides, Annals of the New York Academy of Sciences; NYAS: New York, 1993.

12. Ito, A. S.; Castrucci, A. M. L.; Hruby, V. J.; Hadley, M. E.; Krajcarski, D. T.; Szabo, A. G. Biochemistry 1993, 32, 12264.

13. Taylor, J. W.; Kaiser, E. T. Pharmacol Rev 1986, 38, 291; Taylor, J. W.; Kaiser, E. T. Meth Enzymol 1987, 154, 473.

14. Finer-Moore, J.; Bazan, J. F.; Rubin, J.; Stroud, R. M. In: Fasman, G. D., ed. Prediction of Protein Structure and the Principles of Protein Conformation; Plenum Press: New York, 1989.

15. Branden, C.; Tooze, J. Introduction to Protein Structure; Garland: New York, 1991.

16. Rosenblatt, M.; Beaudette, N. V.; Fasman, G. D. Proc Natl Acad Sci USA 1980, 77, 3983.

17. Emr, S. D.; Silhavy, T. J. Proc Natl Acad Sci USA 1983, 80, 4599.

18. Brigs, M. S.; Cornell, D. G.; Dluhy, R. A.; Gierasch, L. M. Science 1986, 233, 206.

19. Gierasch, L. M. Biochemistry 1989, 28, 923.

20. Bruch, M. D.; Gierasch, L. M. J Biol Chem 1990, 265, 3851; Bruch, M. D.; McKnight, C. J.; Gierasch, L. M. Biochemistry 1989, 28, 8554.

21. Halgren, T. A. Curr Opin Struct Biol 1995, 5, 205; van Belle, D. Computer Studies of Electronic Polarization Effects in Biological Systems; Thesis, Université Libre de Bruxelles, Belgium, 1992.

22. Pethig, R. Dielectric and Electronic Properties of Biological Materials; Wiley: New York; 1979.

23. Gilson, M. K. Curr Opin Struct Biol 1995, 5, 216.

24. Friedman, H. L. Mol Physiol 1975, 29, 1533.

25. Rogers, N. K. In: Frasman, G. D., ed. Prediction of Protein Structure and the Principles of Protein Conformation; Plenum: New York, 1989.

26. Wiberg, K. B. J Am Chem Soc 1965, 87, 1070.

27. Allen, M. P.; Tildesley, D. J. Computer Simulation of Liquids; Oxford University Press: Oxford, 1987.

28. Wang, Z.; Jones, J. D.; Rizo, J.; Gierasch, L. M. Biochemistry 1993, 32, 13991.

29. Jones, J. D.; McKnight, C. J.; Gierasch, L. M. J Bioenerg Biomembr 1990, 22, 213.

30. Landry, S. J.; Gierasch, L. M. Trends Biochem Sci 1991, 16, 159.

31. McKnight, C. J.; Rafalsk, M.; Gierasch, L. M. Biochemistry 1991, 30, 6241.

32. Jones, J. D.; Gierasch, L. M. Biophys J 1994, 67, 1534. 